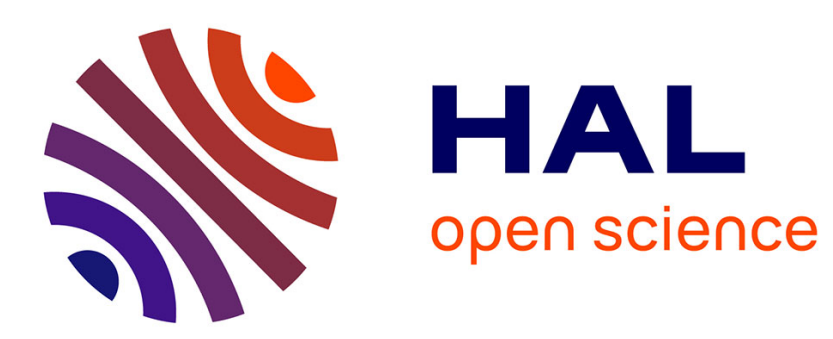

\title{
Aspects pragmatiques de la négation renforcée en italien
} Danièle Godard, Jean-Marie Marandin

\section{To cite this version:}

Danièle Godard, Jean-Marie Marandin. Aspects pragmatiques de la négation renforcée en italien. Floricic, Franck;. La négation dans les langues romanes, John Benjamins, pp.136-160, 2007, Linguisticae Investigationes Supplementa. halshs-00748425

\section{HAL Id: halshs-00748425 \\ https://shs.hal.science/halshs-00748425}

Submitted on 25 Nov 2012

HAL is a multi-disciplinary open access archive for the deposit and dissemination of scientific research documents, whether they are published or not. The documents may come from teaching and research institutions in France or abroad, or from public or private research centers.
L'archive ouverte pluridisciplinaire HAL, est destinée au dépôt et à la diffusion de documents scientifiques de niveau recherche, publiés ou non, émanant des établissements d'enseignement et de recherche français ou étrangers, des laboratoires publics ou privés. 


\title{
Aspects pragmatiques de la négation renforcée en italien
}

\author{
Danièle Godard et Jean-Marie Marandin \\ CNRS et Université Paris 7
}

\section{Introduction*}

Le système général de la négation en italien met en jeu l'adverbe négatif non, qui apparait immédiatement avant le verbe ${ }^{1}$, et des mots appelés habituellement " mots-n » («n-words ») à la suite de I. Laka (1990). Les mots-n sont des formes qui peuvent soit induire une interprétation négative pour la phrase, soit avoir une interprétation d'indéfinis et / ou fonctionner comme des items de polarité négative, en fonction du contexte linguistique dans lequel ils apparaissent. Nous considérons ici les mots-n italiens nessuno ('personne', 'aucun'), niente et nulla ('rien'), dans le contexte d'une phrase indépendante, ou phrase racine. Ces mots-n ne se comportent pas de la même manière suivant qu'ils se trouvent en position préverbale ou postverbale ; une asymétrie comparable existe en espagnol et en portugais, et aussi en catalan, bien qu'elle y soit moins claire (T. Espinal 2006). Lorsque ces mots apparaissent avant le verbe, l'adverbe de la négation phrastique non est absent ; lorsqu'ils apparaissent après le verbe, non est obligatoire $^{2}$ :

(1) a. Paolo non viene

Paolo NEG vient

'Paolo ne vient pas'

b. Nessuno viene / *Nessuno non viene

Personne vient / personne NEG vient

'Personne ne vient'

c. Paolo non vede nessuno / *Paolo vede nessuno

Paolo NEG voit personne / Paolo voit personne

'Paolo ne voit personne'

A côté des données illustrées en (1), il existe des phrases où, de fait, un mot-n et non co-existent en position préverbale en italien. Le premier cas met en jeu un non accentué : on a alors une interprétation avec double négation (2).

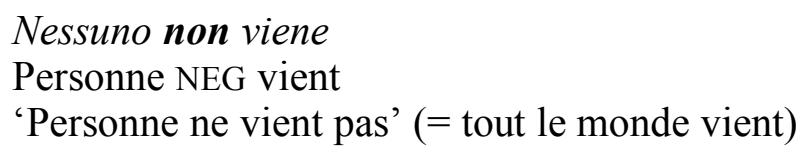

Deux autres cas sont signalés dans les chapitres sur l'ordre des mots et sur la négation de la grande grammaire de L. Renzi et al. (P. Benincà et al. 1998, E. Manzotti et L. Rigamonti 1991), qui, à l'inverse de (2) donnent lieu à une interprétation avec négation

\footnotetext{
* Nous remercions particulièrement pour leurs jugements et leurs suggestions Alda Mari, Lucia Tovena, et Marta Donazzan, ainsi que nos deux relecteurs. Nos données ont été obtenues par élicitation de jugements de félicité auprès d'informateurs qui sont tous du nord de l'Italie. Nous ne faisons donc aucune hypothèse en ce qui concerne les autres régions.

1 Non peut cependant être séparé du verbe par sempre, voir (7a).

2 Les exemples sont soit glosés soit traduits (entre guillements); nous ne chercherons pas à rendre l'effet pragmatique des énoncés italiens en français.
} 
unique, appelée concordance négative. En (3), on dit qu'il faut une certaine 'distance' entre le mot-n et non, c'est-à-dire que le mot-n fait partie d'un SN complexe (voir F. Floricic 2005). En (4), la phrase comporte un constituant initial qui est le lieu d'une prosodie spécifique, qui demanderait une analyse de détail que nous n'entreprendrons pas ici ; il semble cependant qu'il s'agisse d'un contour particulier localisé sur le dernier mot de ce syntagme initial (qui peut ne pas correspondre au mot-n), et que nous notons avec des majuscules. Dans l'un et l'autre cas, les acceptabilités sont sujettes à variation, au sens où tous les locuteurs de l'italien n'ont pas ces possibilités, d'où la marque '\%' devant les exemples. On gardera en mémoire que la seule interprétation pertinente est celle qui ne comporte qu'une seule négation logique.

(3)a. \% Da nessuna parte non potrebbe star meglio

'Nulle part, ça ne pourrait être mieux'

b. \% Nessuna delle piante non sembra malata

'Aucune plante n'a l'air malade'

b'. *Nessuna non sembra malata

Aucune NEG n'a l'air malade

'Aucune n'a l'air malade'

(4)a. \% NESSUNO non è venuto!

Personne NEG est venu

'Personne n'est venu'

b. \% NIENTE non ho fatto

Rien NEG ai fait

'Je n'ai rien fait'

c. $\%$ A nessuno degli STUDENTI non ha parlato

A aucun des étudiants NEG a parlé

'A aucun des étudiants il n'a parlé'

La situation illustrée en (1) a donné lieu à de nombreuses analyses, qui comportent un choix sur plusieurs points: (i) quel est le statut des mots-n (des négations, c'est-à-dire des quantifieurs négatifs, ou bien des indéfinis, ou bien des mots ambigus entre les deux analyses) ${ }^{3}$ ? (ii) comment rendre compte de l'asymétrie illustrée en $(1 b, c)$, et de la concordance négative dans le cas où les mots-n sont postverbaux (où l'interprétation de Paolo non vede nessuno est celle d'une négation unique, bien que, pris séparément, non (1a), et les mots-n (1b) puissent fonctionner comme des négations)?

Nous laissons de côté la discussion de ces questions complexes, car notre objet d'étude est connexe. Nous nous intéressons ici en effet aux cas illustrés en (4), qui n'ont pas été étudiés de manière précise, et pour lesquels nous proposons l'analyse suivante. Il $\mathrm{y}$ a deux systèmes de négation en italien : la négation « ordinaire » illustrée en (1), et la négation « renforcée » illustrée en (4), qui ont des propriétés formelles et pragmatiques différentes.

(a) D'un point de vue pragmatique, la négation renforcée correspond à l'un des cas de négation métalinguistique (refus par le locuteur d'une proposition présente explicitement ou implicitement dans le contexte), usage de la négation qui s'oppose à la négation descriptive (description d'une situation qui ne correspond pas aux conditions de vérité de la phrase positive), dans les termes de L. Horn (1989). La négation

\footnotetext{
3 Les mots-n italiens sont, par exemple, analysés comme des quantifieurs dans R. Zanuttini (1991), F. Corblin et L. Tovena (2003); comme des indéfinis dans R. Rizzi (1982), P. Acquaviva (1997), A. Przepiórkowski (1999); comme des mots ambigus dans A. Giannakidou (2002).
} 
ordinaire peut être employée soit comme négation descriptive soit comme négation métalinguistique.

(b) La co-occurrence du mot-n et de non devant le verbe est permise par l'annulation, dans les énoncés qui expriment une dénégation, des contraintes qui aboutissent aux faits illustrés en $(1 \mathrm{~b}, \mathrm{c})$, quelle que soit la façon dont on les représente dans la grammaire.

(c) La construction (4) repose sur une construction qui existe indépendamment de la négation.

Dans la section 2, nous décrivons les propriétés formelles qui caractérisent les phrases de (4). Nous montrons, en particulier, que le constituant initial a des fonctions grammaticales variées; la construction est donc identifiée par la conjonction d'une propriété de linéarisation d'un constituant avec un certain contour prosodique, plutôt que par une structure syntaxique. On retrouve ce fonctionnement dans des phrases positives avec un constituant initial, comme dans des phrases négatives sans redoublement de la négation préverbale. Dans la mesure où celles-ci ont été caractérisées en termes de structure informationnelle, nous regardons les propriétés informationnelles associées à l'ensemble de ces phrases (positives et négatives) dans la section 3. Nous montrons que, contrairement à ce qui a été avancé, il n'y a pas de corrélation unique entre le constituant initial et un rôle spécifique dans l'articulation informationnelle. En revanche, les énoncés de la forme de (4) ont en commun la propriété pragmatique d'être des dénégations d'une proposition, ce qui est un des souscas de la négation métalinguistique (voir B. Geurts 1998). Dans la section 5, nous relions le redoublement de la négation de (4) à une hypothèse générale concernant la négation renforcée. Nous reprenons l'interprétation que P. Kiparsky et C. Condoravdi (2006) donnent du cycle de Jespersen : le renforcement de la négation est motivé par le besoin d'exprimer la négation métalinguistique par une différence formelle. Nous avançons que ce renforcement peut se faire par le recyclage de matériel négatif, dans des conditions syntaxiques qui violent celles de la négation ordinaire. Enfin, dans la dernière section, nous proposons une modélisation de cette analyse dans un modèle dialogique proposé par J. Ginzburg (en prép) : elle utilise des structures de traits, et s'intègre dans le formalisme de la grammaire syntagmatique HPSG, comme une modélisation de la partie 'contexte' des énoncés.

\section{Les propriétés formelles des phrases en (4)}

Les phrases de (4) mettent en jeu, d'une part, l'adverbe non, et d'autre part, un constituant initial qui comporte un mot-n. Nous les examinons dans cet ordre.

\subsection{L'adverbe non}

Nous analysons non comme un adverbe ajout au verbe lexical, ou à une coordination de verbes lexicaux (A. Abeillé et D. Godard 2003) ${ }^{4}$. La raison qui empêche d'analyser non comme un ajout au SV est qu'il n'a pas portée sur une coordination de SV, c'est-à-dire de verbes avec leurs compléments, bien qu'il ait portée sur une coordination de verbes lexicaux (voir aussi J-B. Kim 2000). En (5), non autorise nessun giornale, qui est le complément de la coordination des V compra et legge ou comprare et leggere. A l'inverse, les exemples de (6) illustrent une coordination de SV, dont le second membre contient un mot-n. Dans ces exemples, non, qui apparaît devant le premier V, ne peut

\footnotetext{
4 Une analyse alternative de non est donnée par J-B. Kim (2000), où c'est une « tête faible » qui prend comme complément un verbe dont il hérite la catégorie (sur les têtes faibles, voir J. Tseng 2002).
} 
pas autoriser le mot-n dans le second SV, ce qui montre qu'il n'a portée que sur le premier membre de la coordination ${ }^{5}$.

(5)a. Paolo non compra o legge nessun giornale

'Paul n'achète ou ne lit aucun journal'

b. Paolo non sembra comprare o leggere nessun giornale

'Paul ne semble acheter ou lire aucun journal'

(6)a. *Paolo non legge giornali e / o guarda nessuna notizia in televisione

Paolo NEG lit de journaux et / ou regarde aucun journal télévisé

'Paolo ne lit pas de journaux et / ou ne regarde aucun journal télévisé'

b. *Paolo sembra non leggere giornali o guardare nessuna notizia in televisione

Paolo semble NEG lire de journaux ou regarder aucun journal télévisé

c. *Non comprando giornali e / o guardando nessun programma in televisione,

Paolo vive fuori dal mondo

NEG achetant de journaux et regardant aucun programme télévisé,

Paolo vit hors du monde

Un mot-n postverbal est autorisé s'il est dans la portée de non (1). On voit que cela est possible si non précède une coordination de $\mathrm{V}$, et impossible si non précède une coordination de SV. Le contraste est expliqué si non est analysé comme un ajout à un $\mathrm{V}$ lexical ou à une coordination de Vs lexicaux. Dans les termes de A. Abeillé et D. Godard (2000, 2006), qui font usage d'une propriété syntaxique de poids associée aux mots et aux syntagmes, non est un adverbe qui s'adjoint à gauche d'un verbe léger. Les coordinations de V lexicaux sont légères, alors que les SV d'une manière générale sont non-légers. En italien (comme en français), les coordinations et les adjonctions ne comportant que des éléments légers peuvent être légères.

Cette analyse est confortée par le fait que non ne peut pas être séparé du verbe par un autre adverbe : la plupart des adverbes (en particulier les adverbes en -mente) sont exclus. Certains locuteurs, néanmoins, acceptent sempre (J.-B. Kim 2000). Le contraste entre (7b) et (7c) montre que c'est la position de l'adverbe volentieri entre non et le verbe qui exclut la phrase, et non pas le fait que cet adverbe ne puisse pas suivre non.

(7)a. \% Non sempre la facciamo, ma vale la pena di continuare a richierdercela

'Nous ne la faisons pas toujours, mais ça vaut la peine de continuer à nous la demander' (exemple de J.-B. Kim (2000))

b. * Gianni non volentieri vedrà domani

Gianni NEG volontiers viendra demain

c. Gianni non vedrà volentieri domani

Gianni NEG viendra volontiers demain

'Gianni ne viendra pas volontiers demain'

Ces données sont attendues si les adverbes, d'une manière générale, ne s'adjoignent pas à un constituant verbal léger, ou s'ils le font, rendent ce constituant non-léger. En revanche, l'adverbe non s'adjoint à une catégorie verbale légère (le V lexical, ou une

\footnotetext{
5 Le mot-n est autorisé en (i) ci-dessous par la conjonction de coordination né qui, elle-même, comporte une négation sémantique :

(i) Paolo non legge giornali né guarda nessuna notizia in televisione

Paolo NEG lit de journaux ni regarde aucun journal télévisé

'Paolo ne lit pas de journaux ni ne regarde aucun journal télévisé'
} 
coordination de $\mathrm{V}$ lexicaux, ou un syntagme tête-ajout léger). Nous admettrons que sempre, étant lui aussi un adverbe léger (ou pouvant être léger), peut s'adjoindre au V lexical, et former avec lui un syntagme léger, auquel non peut s'adjoindre (7a). La structure de (6a) est donnée en (8) : nessuna notizia est hors de la portée de non.

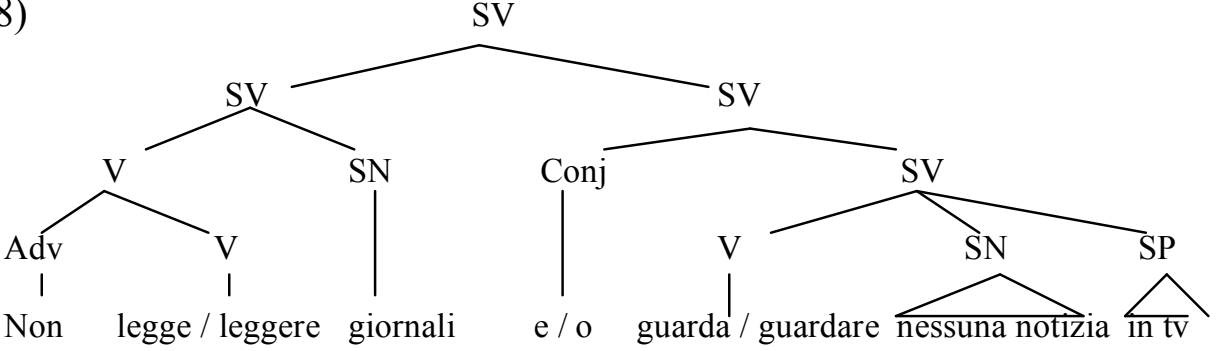

Nous adoptons ici la grammaire syntagmatique HPSG (voir C. Pollard et I. A. Sag 1994, I. A. Sag et al. 2003, A. Abeillé 2006). Si l'on s'en tient aux propriétés syntaxiques de non, on a la description en (9). Comme les adverbes d'une manière générale, non peut s'adjoindre à une tête. Le trait MOD précise les propriétés du constituant auquel il peut s'adjoindre : il s'agit d'un verbe léger. Cette spécification lexicale (via le trait MOD) lui permet de fonctionner comme un ajout selon le schéma général tête-ajout donné en (12b).

(9) Description syntaxique de l'adverbe non

[CAT I TETE adverbe [MOD verbe [POIDS léger $]]]$

\subsection{Le constituant initial}

Les phrases de (4) ont en commun d'avoir un constituant initial caractérisé par deux propriétés formelles : une propriété d'ordre (il est à l'initiale de la phrase) et une propriété prosodique : il porte un contour spécifique, ancré sur sa frontière droite. Ce contour ne peut être précisé sans une étude phonétique sérieuse qui demande encore à être faite; nous nous contentons donc de le nommer «contour spécifique », et nous le représentons en mettant en majuscules le mot qui est le support du contour. Nous insistons sur le fait que ce contour est ancré sur le dernier mot du syntagme, et non pas sur le mot-n lui-même. Ainsi, en (4c), c'est le $\mathrm{N}$ tête du complément (studenti) qui le porte, et non le n-word (nessuno) qui est la tête du constituant initial complet. En dehors de ces propriétés positionnelle et prosodique, les constituants initiaux de (4) n'ont pas de propriété formelle commune; en particulier, ils ont des fonctions syntaxiques différentes.

On peut montrer que le SP initial de (4c), repris ici en (10a), est extrait : il met en jeu une relation d'extraction avec un constituant initial et un " gap » correspondant dans la phrase elle-même. En effet, ni sa forme ni sa fonction ne correspondent à celles d'un sujet, ce sont celles d'un complément du V. Il en va de même en (4b), repris en (10b), ou en (10c) :

(10)a. \% A nessuno degli STUDENTI non ha parlato

b. \% NIENTE non ho fatto! (Rien j'ai fait)

c. \% Con NESSUNO non ama parlare qui

Avec personne NEG il aime parler ici 
De plus, comme on le voit en (10c), il peut y avoir une certaine distance entre le V dont il est complément et la position du constituant extrait: le SP con nessuno est complément de parlare, qui est lui-même le verbe tête du SV à l'infinitif complément de $a m a$.

En revanche, il n'y a pas d'argument qui permettrait de soutenir que le constituant initial est extrait s'il est sujet ou ajout à la phrase, comme on le voit en (4a), repris en (11a) ou en (11b).

(11)a. \% NESSUNO non è venuto

b. \% Da nessuna PARTE, non ho visto Paolo

'Nulle part je n'ai vu Paolo'

On admettra donc que les constituants initiaux de (11) ne se distinguent pas syntaxiquement de ce qu'ils sont dans une phrase ordinaire, c'est-à-dire sans la présence du contour marqué et de l'adverbe non : ils sont respectivement sujet et ajout à la phrase.

\subsection{Modélisation}

Dans le modèle de la grammaire syntagmatique HPSG, les constituants initiaux de (4) correspondent à la branche non-tête dans trois constructions différentes : la construction tête-sujet, la construction tête-ajout, et la construction tête-extrait, qui sont donnés en (12). Le trait SYNTAGME décrit les propriétés pertinentes de la construction elle-même, les traits BRANCHE-TETE et BRANCHE-NON-TETE décrivent les traits pertinents des constituants de la construction. L'identité des pointeurs [1] ou [A] indique que la valeur du trait aux différents endroits de la description est identique 6 . Un constituant est défini par la ou les propriétés pertinentes dans la description; par exemple, en (12a), la branche-tête est un constituant qui porte le trait [SUJET $<[1]>$ ], c'est-à-dire qu'il attend un sujet lui-même défini par un ensemble de propriétés syntaxiques et sémantiques noté par [1].

(12) Les constructions syntaxiques pour les phrases de (4)

a. construction tête-sujet $=>$

b. construction tête-ajout $=>$

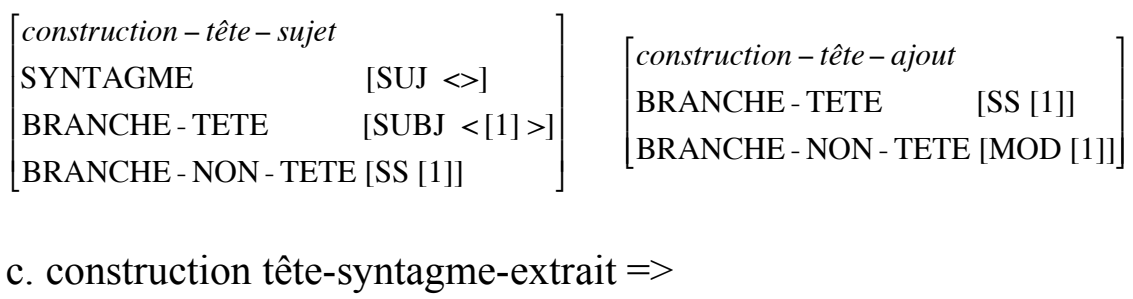

$\left[\begin{array}{ll}\begin{array}{l}\text { construction - tête-extrait } \\ \text { SYNTAGME }\end{array} & {[\text { SLASH }[\mathrm{A}]]} \\ \text { BRANCHE-TETE } & {\left[\begin{array}{l}\text { TETE verbe } \\ \text { SLASH }\{[1]\} \cup[\mathrm{A}]\end{array}\right]} \\ \text { BRANCHE-NON-TETE }[\mathrm{LOC}[1]]\end{array}\right]$

6 «<>» note les listes, « $\oplus »$ la concaténation de listes, «\{\} $\}$ les ensembles, et «U» l'union d'ensembles. 
Le schéma (12a) autorise la combinatoire d'un constituant avec certaines propriétés syntaxiques et sémantiques (notées par SS [1]) avec un constituant tête qui attend un sujet ayant les mêmes propriétés, et la construction annule l'attente du sujet. Le schéma (12b) autorise un constituant à se combiner avec un constituant tête dans la mesure où les propriétés syntaxiques et sémantiques de ce dernier correspondent aux spécifications qu'il porte concernant l'adjonction, qui se trouvent dans le trait MOD (l'ajout sélectionne la tête). Enfin, dans le schéma (12c), le constituant tête est de type verbe (a pour tête un verbe), et porte l'indication qu'un constituant au moins a été extrait et ne se trouve donc pas réalisé in situ. Le trait SLASH encode cette extraction depuis le prédicat dont un argument est extrait jusqu'à la phrase qui domine le constituant initial (extrait) dont les propriétés correspondent à celles d'un constituant dans SLASH. La construction têteextrait demande que les propriétés pertinentes (encodées dans le trait LOC) de l'élément extrait et de l'élément dans SLASH soient identiques?

\section{Les conditions de félicité des énoncés de (4)}

Les phrases en (4) se caractérisent par un constituant qui doit être initial et porteur d'un contour spécifique. Cette caractérisation se rencontre également dans des phrases positives. Les phrases qui présentent cette caractérisation, qu'elles soient négatives ou positives, ont des propriétés de félicité particulières communes. Les phrases positives ont été étudiées et on a proposé de recourir à la structure informationnelle pour les caractériser : le constituant initial est analysé comme «focus», ou comme « constrastif », ou comme « focus contrastif » (voir, par exemple, L. Rizzi 1997). Dans cette section, nous montrons que le recours à la structure informationnelle ne permet pas de caractériser la construction, ni quand le constituant est positif ni quand il est négatif. En conséquence, nous changeons de perspective et nous adoptons un cadre dialogique. Nous montrons que ces phrases correspondent à des énoncés qui expriment le rejet d'une proposition (explicite ou implicite) accessible dans le contexte.

\subsection{La partition informationnelle n'est pas unique}

Regardons en premier lieu les phrases positives parallèles à celles de (4) : le constituant initial est-il associé à un statut spécifique dans l'articulation informationnelle de l'énoncé ? En dépit de ce qui est suggéré dans la littérature, on observe aisément qu'il n'est pas associé à un statut informationnel spécifique et unique. Nous adoptons les définitions suivantes. Le « focus informationnel » est le statut du constituant qui apporte l'information nouvelle pertinente dans la progression du discours; il a pour complément le « fond ». Un constituant est « contrastif » (ou plutôt Kontrastif au sens de E. Vallduví et M. Vilkuna 1998), lorsqu'il dénote un élément qui appartient à un ensemble d'alternatives (explicite ou implicite) accessible dans le contexte.

On note tout d'abord que le constituant initial avec contour marqué peut apparaître dans un contexte où un énoncé «all focus » (c'est-à-dire sans partition informationnelle) est approprié. Plus précisément, on doit distinguer deux types de contexte : les contextes où un thème de discours est déjà introduit et les contextes, qu'on appelle " out of the blue », où le locuteur initie un échange ou un thème de discours. Les énoncés que nous considérons sont appropriés en tant qu'énoncé all focus dans le premier type de contexte (13a) et non dans le second (13b.i). Dans les contextes out of the blue, on ne trouve que des énoncés all focus ordinaires, c'est-à-dire à sujet

\footnotetext{
7 Pour une analyse plus précise en HPSG des propriétés formelles de la construction (4), voir Godard \& Marandin (2006).
} 
préverbal non marqué prosodiquement (13b.ii) ou bien à sujet postverbal dans une construction présentationnelle (13b.iii). Nous revenons plus bas sur (13a) : la réponse de A n'est pas une réponse neutre.

(13)a. [A et B se connaissent ; A est en train de raconter comment s'est passé le colloque auquel il vient d'assister]

A. Nanno Moretti ha fatto il suo intervento

'Nanno Moretti a fait son exposé'

B. E poi?

'Et alors?'

A. GIOVANNI ha applaudito fragorosamente.

'Giovanni a applaudi à tout rompre'

b. [A a organisé une conférence, et ne peut être présent à l'ouverture. Il appelle le secrétariat pour savoir comment les choses se passent]
A. Come sta andando?
'Comment ça se passe?
B. i. \# Molti STUDENTI sono venuti
ii. Molti studenti sono venuti
iii. Sono venuti molti studenti
'Beaucoup d'étudiants sont venus'

Dans les énoncés qui présentent une partition informationnelle de leur contenu, on observe que le constituant initial peut appartenir au focus ou bien au fond. C'est un focus informationnel non Kontrastif en (14) : il explicite la réponse à la question portant sur ce constituant. C'est un focus informationnel Kontrastif en (15), puisqu'il choisit un élément dans un ensemble contextuellement introduit. C'est un topique Kontrastif (il appartient au fond) en (16). En (16), B choisit de répondre " matière par matière » à la question de A, ce qui implique que les interlocuteurs A et B savent que l'examen en question comporte un volet syntaxique et un volet sémantique : le constituant la sintassi dans le premier élément de réponse s'oppose à la semantica dans le second ${ }^{8}$.

(14) A. A chi ha parlato Maria per tutta la serata?

'A qui Maria a parlé pendant toute la soirée?'

B. $\quad$ i.\# A suo ex-ragazzo ha parlato (Maria)

ii. A suo ex-RAGAZZO ha parlato (Maria)

'Elle a parlé à son ex-copain'

(15) A. Suo fratello e suo cugino sono appena arrivati. Sai chi inviterà?

'Son frère et son cousin viennent d'arriver. Tu sais qui elle va inviter ?'

B. Suo FRATELLO non inviterà

'Elle n'invitera pas son frère'

(16) A. I tuoi studenti hanno riuscito l'esame?

'Tes étudiants ont réussi l'examen?'

B. La SINTASSI hanno capito, la semantica, invece, non c'è verso!

'La syntaxe ils ont compris, mais la sémantique, c'est une catastrophe'

\footnotetext{
8 Les constituants la sintassi et la semantica sont des « S-topics» au sens de D. Büring 1997. Ils indiquent comment construire les sous-topiques permettant d'élaborer le thème de discours introduit par la question.
} 
Comme on peut le constater, le constituant initial peut être un focus (en (14) ou (15)), ou appartenir au fond (en (16)). Il peut être Kontrastif (en (15) ou (16)), ou non (en (14)). Il est donc clair que, bien que les phrases de (4) puissent présenter une partition informationnelle de leur contenu, le constituant initial n'est pas associé à un statut informationnel particulier, qui serait caractéristique de la construction.

Ce qui est crucial, c'est que, dans aucun de ces cas, la réponse n'est pas une simple réponse. Les informateurs associent l'usage de ces énoncés à l'expression d'une attitude du locuteur : le locuteur exprime un étonnement à propos de ce qu'il asserte, c'est étonnant pour lui ou bien ce devrait être une cause d'étonnement pour son interlocuteur. Il y a donc quelque chose de plus que le fait de répondre à l'interlocuteur, le contenu asserté ou le fait d'asserter et ce que ça implique d'engagement pour le locuteur. Dans les trois configurations ci-dessus, le locuteur s'oppose à des représentations que les informateurs reconstruisent comme étant associées à la question. En (14), B présente le fait que Marie parle à son ex-copain comme surprenant pour lui ou comme devant surprendre A. Il en est de même en (15) et (16).

Tournons-nous maintenant vers les phrases avec constituant initial négatif. On constate que ce dernier peut remplir les mêmes rôles informationnels que sa contrepartie non négative. Ainsi, il est focus informationnel non Kontrastif en (17), focus Kontrastif en (18) ou (19), et topique Kontrastif en (20). Nous donnons ici à la fois des exemples sans non et avec non (négation renforcée). Comme on le voit, la présence de non est soumise à variation, mais ne change pas les conditions de félicité de l'énoncé.

(17) A. A chi non ha parlato Maria per tutta la serata?

B. i. \# A nessuno degli studenti ha parlato

ii. A nessuno degli STUDENTI ha parlato

iii. \# A nessuno degli studenti non ha parlato

iv. \% A nessuno degli STUDENTI non ha parlato

(18) A. Chi non ha fatto i suoi compiti?

'Qui n'a pas fait ses devoirs?'

B. i. \#Nessuno li ha fatti

ii. NESSUNO li ha fatti

iii. \# Nessuno non li ha fatti

iv. \% NESSUNO non li ha fatti

'Personne ne les a faits'

(19) A. Suo fratello e suo cugino sono appena arrivati. Sai chi inviterà?

B. $\%$ Nessuno di DUE non inviterà

'Elle n'invitera aucun des deux'

(20) A. I tuoi studenti hanno riuscito l'esame?

B. \%Nessuna questione di SINTASSI (quei cretini) non sono riusciti a risolvere! Le due questioni di semantica, invece, tutti le hanno risolte

Aucune question de syntaxe, les imbéciles, NEG ILS.ont réussi à

résoudre! Les deux questions de sémantique, en revanche, tous les ont réussies

Enfin, on observe la même distribution dans les énoncés all focus qu'en (13) cidessus, repris ici en (21b), et dans les mêmes contextes. Le constituant marqué peut apparaître dans un énoncé all focus (21a), mais pas dans un énoncé all focus initiateur de dialogue (21b). La réponse de A en (21a) est associée de façon forte à l'idée que A 
ou B, ou les deux, s'attendaient à ce qu'un exposé de Nanni Moretti soit accueilli par des applaudissements.

(21)a.

A. Nanno Moretti ha fatto il suo intervento

B . E poi, cos'è successo?

A . \% NESSUNO non ha applaudito

b.

A. Come sta andando?

B. i. \# NESSUNO non è venuto

ii. Non è (ancora) venuto nessuno

On observe donc une nouvelle fois qu'il n'y a pas de corrélation entre le constituant initial et un rôle particulier dans l'articulation informationnelle. De plus, ce constituant n'est pas nécessairement Kontrastif. En revanche, on observe le même effet supplémentaire que l'on a vu avec les phrases positives, et cet effet est constant : le locuteur présente le contenu de son énoncé comme étonnant, inattendu. C'est donc de ce côté-là qu'il faut chercher ce qui caractérise la construction 9 .

\subsection{Les énoncés en (4) sont des dénégations de propositions}

Regardons plus particulièrement les phrases négatives de (4), qui comportent un constituant initial négatif avec un contour marqué et l'adverbe non. Comme nous l'avons $\mathrm{vu}$, ils ne sont caractérisés ni par une syntaxe uniforme, ni par une structure informationnelle uniforme, et ils partagent leur structure syntaxique et leur structure informationnelle avec des phrases qui ne comportent pas d'expressions négatives. Enfin, que les énoncés soient négatifs ou non, ils sont associés à un effet pragmatique qui semble bien constituer la propriété discursive caractéristique de l'ensemble des énoncés avec constituant initial prosodiquement marqué. Nous précisons dans cette section en quoi consiste cet effet lorsque la phrase comporte les deux négations préverbales.

L'énonciation des phrases de (4) constitue toujours la dénégation d'une proposition qui est accessible dans le contexte et que nous appelons "proposition cible $»^{10}$. Il ne suffit pas de poser que la proposition cible appartient au "common ground» (R. Stalnaker 1974) : elle doit être " ancienne dans le discours » (selon la terminologie d'E. Prince 1981 : discourse old) et « activée », c'est-à-dire introduite dans l'univers local du dialogue ${ }^{11}$. On peut distinguer deux cas, selon que la proposition est explicitement exprimée dans le contexte, et, plus précisément, dans le tour dialogique précédent, ou bien qu'elle est implicite, au sens où elle est inférable d'un énoncé ou de l'énonciation d'un énoncé dans le contexte immédiat. Le premier cas est illustré en (22) et (23).

A. $\quad$ Pietro ha letto tutti gli scritti di Einstein

'Pietro a lu tous les textes d'Einstein'

\footnotetext{
${ }^{9}$ Nous revenons brièvement dans la section 4 sur le fait que ces énoncés comportent un élément distingué (bien qu'il ne s'agisse pas de partition informationnelle).

${ }^{10}$ L'obligation d'une proposition cible fait penser à l'usage dit « écho » de la négation chez R. van der Sandt (1991) et R. Carston (1996). La notion d'écho est trop générale pour notre propos : la construction que nous étudions met en jeu le contenu d'une proposition, à l'exclusion de tous contenus ancillaires (présuppositions, implicatures etc.), et aussi de la forme, éléments d'information auxquels la notion d'écho est susceptible de s'appliquer.

11 Nous renvoyons aux distinctions de M. Dryer (1996) et à leur discussion dans S. Schwenter (2005) à propos d'une construction analogue en portugais brésilien (voir plus loin).
} 


\section{B. $\quad \%$ Scherzi, NESSUNO (non) ne ha letto \\ 'Tu veux rire, il n'en a lu aucun'}

(23) [Gianni et ses frères sont les voisins de Maria]

A. Per il suo compleanno, Maria inviterà Gianni e i suoi fratelli

'Pour son anniversaire, Maria invitera Gianni et ses frères'

B. $\quad \%$ Nessuno dei suoi VICINI la poveretta (non) inviterà

'La pauvre n'invitera aucun de ses voisins'

Le locuteur B asserte une proposition qui nie directement la proposition assertée par le locuteur ; en (23), le locuteur en change partiellement la formulation en passant de Gianni e $i$ suoi fratelli à suoi vicini. Dans les deux cas, B s'oppose à l'engagement (« commitment») que son interlocuteur manifeste par son énoncé. Cette opposition peut être accompagnée, selon les gloses de nos informateurs, d'une attitude de surprise du locuteur : il est très étonnant de penser que Pietro puisse avoir lu les textes d'Einstein, ou que Maria puisse inviter qui que ce soit parmi ses voisins.

Le second cas est illustré en (24) et en (25) : le locuteur B nie une proposition qui n'est pas explicite, mais contextuellement inférable. Pour que les paquets soient arrivés à destination, encore faut-il qu'ils soient partis, et pour qu'il soit pertinent de demander qui Maria va inviter, encore faut-il qu'elle ait l'intention de faire une invitation.

A. Allora sono arrivati i pacchi?

'Alors, les paquets sont arrivés ?'

B. $\%$ No, NESSUNO non ne è ancora partito!

'Non, aucun n'est encore parti'
A. Sai chi Maria inviterà per il suo compleanno?
'Tu sais qui Maria invitera pour son anniversaire?'
B. $\%$ NESSUNO (Maria) non inviterà
'Elle n'invitera personne'

Dans ces deux derniers cas, l'assertion de B s'oppose à une croyance que B attribue à A. Dialogiquement, cela revient à mettre en doute la pertinence de la question de $\mathrm{A}$, dans la mesure où $\mathrm{B}$ nie la proposition qui justifie la question de $\mathrm{A}$.

On note qu'il y a une différence en ce qui concerne l'acceptabilité de la négation non selon que la proposition cible est explicite ou inferée ${ }^{12}$. Quand la proposition est explicite, il semble que l'adverbe soit optionnel, alors que, lorsqu'elle est implicite, non semble obligatoire.

Pour résumer, l'énoncé négatif de la forme (4) a une proposition cible dans le contexte immédiat, ce qui explique qu'on ne les rencontre pas dans les énoncés initiateurs d'échanges dialogiques (cf. (13) et (21) ci-dessus). Quand la proposition cible est explicite, la proposition négative lui est maximalement identique : c'est le cas en (22) et (23), où le verbe est le même et le sujet dénote la même entité.

\section{La négation renforcée et le cycle de Jespersen}

Si les phrases de (4) sont bien associées à des conditions pragmatiques constantes - il s'agit de dénégations de propositions - on peut se demander pourquoi cette construction

\footnotetext{
12 Nous nous limitons, bien sûr, aux locuteurs qui ont cette forme de négation à leur disposition.
} 
existe : le même effet existe avec les phrases similaires (avec constituant initial prosodiquement marqué) mais sans non pour les locuteurs qui n'ont pas la négation préverbale redoublée. La réponse nous est ici donnée par une étude de P. Kiparsky et C. Condoravdi (2006), que nous résumons, avant de voir comment leur proposition s'applique au cas qui nous occupe.

P. Kiparsky et C. Condoravdi (2006) étudient le renouvellement périodique des expressions négatives, en prenant l'exemple du grec. Dans un premier temps, la négation ordinaire (dans les termes de Kiparsky et Condoravdi, «négation simple»), est renforcée par l'adjonction d'un indéfini et forme une expression complexe, ou «négation renforcée» (dans les termes de Kiparsky et Condoravdi, «négation emphatique») ${ }^{13}$. Puis, cette expression complexe tend à se comporter comme une négation ordinaire, et le processus se répète par un nouveau renforcement ; c'est ce qui constitue ce que l'on appelle le cycle de Jespersen (1917). Cependant, ces auteurs se séparent de l'analyse de Jespersen, qui avait attribué la raison de l'affaiblissement de la négation à un amuissement phonétique, en s'appuyant notamment sur l'évolution de la négation de phrase dans l'histoire du latin, puis du latin au français, et dans l'histoire du français même. Ils observent, en effet, que le rôle du facteur phonétique n'est absolument pas confirmé par le grec, où le cycle de Jespersen semble connaître pourtant une grande vigueur. C'est l'étude des propriétés pragmatiques de la négation renforcée qui leur fournit le schéma explicatif : l'usage de la négation renforcée est contraint par des facteurs pragmatiques.

Leur proposition est la suivante. Les langues ont deux types de négation, qui associent une forme et un usage : la négation ordinaire, sans contrainte pragmatique, et la négation renforcée, qui est contrainte pragmatiquement. Essentiellement, la négation renforcée sert à des dénégations de proposition. Les deux types de négation correspondent donc, du point de vue pragmatique, à la distinction de L. Horn (1989) entre négation descriptive et métalinguistique (plus précisément, pour ce qui est de la négation métalinguistique, il s'agit des proposition denials dans la typologie des dénégations de B. Geurts 1998). Pour P. Kiparsky et C. Condoravdi, le cycle de Jespersen s'explique par l'interaction de deux forces: d'une part, la nécessité de maintenir le contraste entre deux formes de négation pour des raisons pragmatiques, et d'autre part, l'affaiblissement sémantique de la forme de négation renforcée dû lui-même à cet usage pragmatique. Une fois que la négation renforcée est affaiblie, elle se grammaticalise en négation ordinaire, et on a la reconstruction d'une nouvelle négation renforcée.

P. Kiparsky et C. Condoravdi ont en tête la négation renforcée de la forme « négation ordinaire + indéfini » (comme ne + pas / mie etc. dans l'histoire du français). Notre proposition est la suivante : en plus de l'incorporation d'éléments non négatifs dans le système de la négation, les langues ont une seconde façon de renforcer la négation : le recyclage du matériel négatif. Le matériel négatif recyclé se distingue du matériel négatif de la négation ordinaire par le fait qu'il n'obéit pas aux mêmes contraintes syntaxiques. C'est ce qu'on observe dans les phrases de (4) : l'adverbe phrastique non apparaît avec un mot-n préverbal, ce qui est impossible dans le système de la négation ordinaire (illustré en (1)).

Cette analyse est confirmée par l'existence dans une autre langue romane d'une négation renforcée, qui réutilise du matériel négatif et qui a des conditions pragmatiques comparables. Il s'agit de la négation du portugais brésilien de la forme $n \tilde{a} o \quad V \ldots$ não, étudiée en particulier dans S. Schwenter $(2005,2006)$, alors que la négation descriptive est $n \tilde{a} o \quad V$. La seconde négation viole les contraintes syntaxiques qui régissent la

13 La notion de négation "emphatique" a été critiquée par S. Schwenter (2006). 
première, puisqu'elle apparaît en position post-verbale. Un exemple est donné rapidement en $(26)^{14}$ :

(26) [A comprend que son frère a manqué un programme qu'il voulait voir à la télévision]
A. Você viu esse programa?
'Tu as regardé ce programme?'
B. Não vi não
NEG j'ai vu NEG
'Non, je ne l'ai pas vu'

La création de la négation renforcée qu'on oberve en (4) et dans les exemples discutés dans la section 3 ci-dessus, met donc en jeu les aspects suivants :

- (i) un type de mouvement dialogique par lequel un locuteur refuse d'entériner un contenu propositionnel parmi les contenus qu'il accepte pour le dialogue en cours. Ce mouvement dialogique est associé en particulier avec une certaine construction (définie en termes d'ordre des mots et de contour prosodique).

- (ii) la compatibilité de cette construction avec des phrases positives ou négatives. Lorsqu'il y a une négation, cet effet coïncide avec une négation métalinguistique, c'està-dire la dénégation d'une proposition localement accessible dans le contexte.

- (iii) le renforcement de l'expression de la négation par l'introduction de non, dont la co-occurrence avec une expression préverbale comportant un mot-n viole une contrainte caractéristique de la négation ordinaire.

On notera que les langues ne choissisent pas de façon exclusive entre les deux types de renforcement de la négation. Ainsi, l'italien connaît les deux. D'une part, on a le renforcement de non par un indéfini, ce qui donne l'expression négative non ... mica, dont les conditions pragmatiques ont été décrites d'abord dans G. Cinque (1976), et R. Zanuttini (1997) en termes de présupposition du locuteur, puis reprises dans L. Tovena (2000), et ensuite S. Schwenter (2006) qui montrent que l'énoncé où apparaît non ... mica est une dénégation de proposition. S. Schwenter contraste l'acceptabilité de la réponse dans les deux contextes différents suivants :

(27)a. A. Chi viene a prenderti?

'Qui vient te prendre?'

B. \# Non so. Ma Gianni non ha mica la macchina. Je ne sais pas. Mais Gianni NEG a MICA la voiture

b. A. Chi viene a prenderti, Gianni?

B. Non so. Ma Gianni non ha mica la macchina.

L'occurrence de mica dans la réponse n'est appropriée que dans un contexte qui permet l'activation de la proposition 'Gianni a la voiture'. C'est le cas en (27b) où A propose une réponse possible Gianni, ce qui permet à $\mathrm{B}$ d'attribuer à $\mathrm{A}$ la croyance que Gianni a les moyens de le véhiculer. La réponse de $\mathrm{B}$ revient à refuser la suggestion de réponse faite par A.

A côté de cette négation renforcée par un indéfini, on a la construction que nous étudions ici, par recyclage de matériel négatif, illustrée en (4). Les deux formes de négation renforcée se distinguent selon une autre dimension, que nous avons jusqu'ici laissée de côté : la distinction ou l'absence de distinction d'une partie du contenu dénié.

14 Voir aussi F. Floricic et F. Mignon (2006), pour une analyse de la réduplication de no en italien. 
La construction que nous étudions présente un constituant placé en tête d'énoncé et distingué prosodiquement. Nous proposons de l'analyser comme ce que R. Carston (1996) appelle « le foyer de l'objection » (focus of objection). Bien que, d'un point de vue logique, la négation porte sur la proposition entière, on a observé qu'une partie du contenu de cette proposition peut être distinguée, celle sur laquelle porte spécifiquement le désaccord (R. Carston 1996, B. Geurts 1998). C'est précisément la distinction de cette partie qu'opère notre construction ${ }^{15}$. Par contre, la dénégation opérée dans les énoncés présentant non ..mica porte sur la proposition entière sans distinction de partie.

Nous résumons le contenu de cette section dans le tableau suivant :

\begin{tabular}{|c|c|c|c|}
\hline & \multirow{2}{*}{$\begin{array}{l}\text { Type de la } \\
\text { forme }\end{array}$} & \multicolumn{2}{|c|}{ Usage pragmatique } \\
\hline & & $\begin{array}{l}\text { Négation } \\
\text { descriptive }\end{array}$ & Négation métalinguistique \\
\hline $\begin{array}{l}\text { non } \\
\text { (asymétrie préverbal vs } \\
\text { postverbal pour les } \\
\text { mots-n) }\end{array}$ & $\begin{array}{l}\text { Négation } \\
\text { ordinaire }\end{array}$ & oui & $\begin{array}{l}\text { Oui } \\
\text { (dénégation de proposition, de } \\
\text { présupposition, d'implicature et } \\
\text { correction) }\end{array}$ \\
\hline non ... mica & $\begin{array}{l}\text { Négation } \\
\text { renforcée }\end{array}$ & non & $\begin{array}{l}\text { - Dénégation de proposition } \\
\text { - Sans partition du contenu }\end{array}$ \\
\hline $\begin{array}{l}\text { Mot-n ...non } \\
\text { (pas d'asymétrie } \\
\text { préverbal vs postverbal } \\
\text { pour les mots-n) }\end{array}$ & $\begin{array}{l}\text { Négation } \\
\text { renforcée }\end{array}$ & non & $\begin{array}{l}\text { - Dénégation de proposition } \\
\text { - Avec partition du contenu }\end{array}$ \\
\hline
\end{tabular}

\section{Modélisation dialogique}

Nous proposons ici une description de la négation métalinguistique qui caractérise la construction (4), dans un modèle du dialogue proposé par J. Ginzburg (2004), légèrement modifié par J-M. Marandin (2005) et O. Bonami et D. Godard (2006) ${ }^{16}$. Ginzburg modélise la dynamique des échanges dialogiques comme un jeu dont les étapes sont enregistrées sur un tableau de jeu («dialogue gameboard»). Chaque participant a un tableau de jeu, qui comporte deux parties, une partie publique et une partie privée. La partie publique comporte les engagements du locuteur (voir le trait ENGT-LOC), de l'interlocuteur (voir le trait ENGT-INTERL), les questions en cours (voir le trait QUD), et la représentation du dernier tour dialogique (DERNIER-TOUR). Parmi les questions en cours, on distingue celle qui est maximale (qui a à voir avec le dernier tour dialogique), ou max-qud. La partie privée enregistre le but de l'énoncé, et le fond, qui distingue entre les propositions qui sont «topiques», et celles qui ne le sont pas; les premières sont en plus scindées selon qu'elles sont topiques pour le locuteur ou pour l'interlocuteur (plus exactement, pour l'interlocuteur selon la représentation que se fait

15 Dans la mesure où il s'agit bien de distinguer une partie dans le contenu de l'énoncé, on a pu confondre cette structuration avec celle de la structure informationnelle, mais les deux partitions sont distinctes. Certaines langues peuvent les marquer de façon identique (c'est vraisemblablement le cas en anglais), et d'autres recourir à des moyens formels distincts : c'est le cas de l'italien ou du portugais brésilien.

16 O. Bonami et D. Godard élaborent le trait FACTS de J. Ginzburg, en le réinterprétant en termes d'engagements des participants (ce à quoi les participants se sont publiquement engagés au cours du dialogue), et en distinguant les deux participants. J.-M. Marandin élabore la partie FOND de la partie privée. 
le locuteur de ce qui est topique pour son interlocuteur). Il y a une relation entre les propositions topiques (qui sont donc enregistrées dans la partie privée du tableau de jeu), et la question max-qud (qui est enregistrée dans la partie publique). Une proposition est topique quand elle est pertinente pour la question max-qud. Les deux parties du tableau de jeu sont représentées comme suit en utilisant des structures de traits $^{17}$.

(28) Les deux composantes du tableau de jeu d'un participant

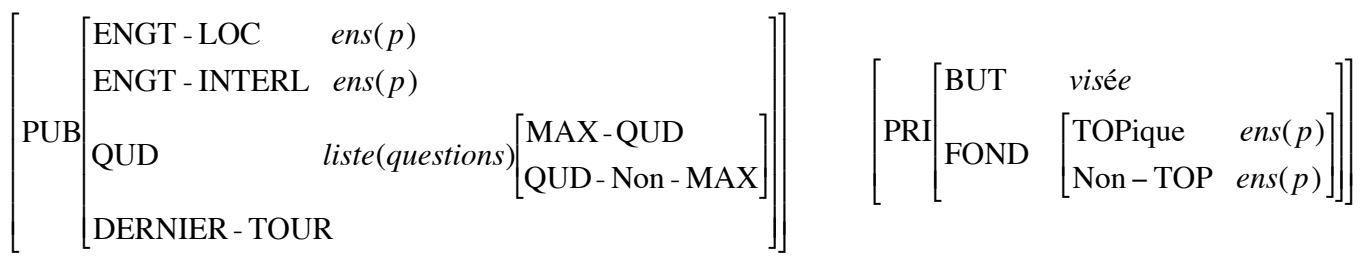

Dans une grammaire HPSG, le tableau de jeu fait partie du contexte d'un énoncé (trait CONTEXTE). Le dernier tour est lui-même décrit en utilisant un tableau de jeu. Nous représentons directement l'effet de l'énoncé sur le tableau de jeu dialogique ${ }^{18}$. Par exemple, la représentation d'un énoncé assertif ou assertion est comme en (29).

(29) Représentation de l'énoncé assertif dans la grammaire

$$
\begin{aligned}
& \text { énoncé assertif }=>\left[\text { CONTEXTE }\left[\begin{array}{l}
\text { assertion } \\
\text { TABLEAU - DIAL } \left.\left[\begin{array}{ll}
\text { PUB } & {[. . .]} \\
\text { PRI } & {[. . .]}
\end{array}\right]\right]
\end{array}\right]\right. \\
& \text { [CONTENU proposition] }
\end{aligned}
$$

Pour rendre la lecture plus facile, nous isolons la représentation du tableau de jeu, et nous sortons la représentation du dernier tour de la structure du tour lui-même, en distinguant entre l'input (le dernier tour) et l'output (le tour dialogique) ${ }^{19}$. La représentation des actes de langage dont nous avons discuté dans cette étude est comme suit. Lorsque le locuteur A asserte une proposition $p$, il la met dans ses engagements (publics); en même temps il met la question polaire correspondante au sommet des questions en discussion (c'est la question max-qud). Si l'interlocuteur B accepte $p$, il met $p$ dans ses propres engagements, et la question portant sur $p$ est retirée de la liste constitutive de QUD.

\footnotetext{
17 Les valeurs des traits en (28) précisent les types; ens(p) dit que la valeur du trait est un ensemble de propositions, liste(questions) que la valeur est une liste de questions.

18 En cela, nous nous éloignons légèrement de la représentation de J. Ginzburg et I.A. Sag (2000), voir O. Bonami et D. Godard (2006).

19 Dans une grammaire compacte, le contexte comprendrait à la fois le tableau dialogique correspondant à l'énoncé et le tableau dialogique correspondant au tour précédent.
} 
(30) Tableau de jeu du locuteur quand il asserte $p$ (O. Bonami et D. Godard 2006) :

$\left[\mathrm{PUB}\left[\begin{array}{ll}\text { ENGT }- \text { LOC } & {[\mathrm{S}]} \\ \text { ENGT - INTERL } & {[\mathrm{A}]} \\ \text { QUD } & {[\mathrm{Q}]}\end{array}\right] \approx\left[\begin{array}{ll}\text { PUB }\left[\begin{array}{ll}\text { ENGT - LOC } & \{\mathrm{p}\} \cup[\mathrm{S}] \\ \text { ENGT - INTERL } & {[\mathrm{A}]} \\ \text { QUD } & <\mathrm{p} ?>\oplus[\mathrm{Q}]\end{array}\right]\end{array}\right]\right.$

(31) Tableau de jeu du locuteur quand il accepte $p$ asserté par l'interlocuteur (O. Bonami et D. Godard 2006) :

$\left[\mathrm{PUB}\left[\begin{array}{ll}\text { ENGT - LOC } & {[\mathrm{S}]} \\ \text { ENGT - INTERL } & \{\mathrm{p}\} \cup[\mathrm{A}] \\ \mathrm{QUD} & <\mathrm{p} ?>\oplus[\mathrm{Q}]\end{array}\right] \approx>\left[\mathrm{PUB}\left[\begin{array}{ll}\text { ENGT - LOC } & \{\mathrm{p}\} \cup[\mathrm{S}] \\ \text { ENGT - INTERL }\{\mathrm{p}\} \cup[\mathrm{A}] \\ \mathrm{QUD} & {[\mathrm{Q}]}\end{array}\right]\right]\right.$

Le locuteur peut également nier une proposition assertée par l'interlocuteur (ce qui est l'une des formes de dénégation de proposition). Quelle est alors la différence avec la réponse négative à une question (A. Marie est-elle venue? B. Non, elle n'est pas venue). Elle ne réside pas dans le résultat (le locuteur s'engage sur non- $p$ ), mais dans l'avancement du dialogue. Alors que la réponse à la question totale est congruente, et manifeste un avancement sans heurts, en quelque sorte linéaire, la dénégation n'a pas cette propriété ; c'est un acte qui a un coût dialogique, et se justifie lorsqu'il est associé, comme nous l'avons vu pour la construction (4), à l'inattendu de l'assertion de $p$. On contraste la réponse négative à la question totale (32) avec la dénégation (33) :

(32) Tableau de jeu du locuteur quand il répond non- $p$ à la question $p$ ? de l'interlocuteur :

$\left[\begin{array}{ll}\text { PUB }\left[\begin{array}{ll}\text { ENGT - LOC } & {[\mathrm{S}]} \\ \text { ENGT - INTERL } & {[\mathrm{A}]} \\ \mathrm{QUD} & <p ?>\oplus[\mathrm{Q}]\end{array}\right]\end{array}\right] \approx\left[\begin{array}{ll}\mathrm{PUB}\left[\begin{array}{ll}\text { ENGT - LOC } & {[\mathrm{S}] \cup\{\neg p\}} \\ \mathrm{QUD} & <\neg p ?>\oplus[\mathrm{Q}]\end{array}\right]\end{array}\right]$

(33) Tableau de jeu du locuteur quand il nie $p$ asserté par l'interlocuteur :

$\left[\mathrm{PUB}\left[\begin{array}{ll}\text { ENGT - LOC } & {[\mathrm{S}]} \\ \text { ENGT - INTERL } & \{p\} \cup[\mathrm{A}] \\ \mathrm{QUD} & <p ?>\oplus[\mathrm{Q}]\end{array}\right]\right] \approx>\left[\mathrm{PUB}\left[\begin{array}{ll}\text { ENGT - LOC } & {[\mathrm{S}] \cup\{\neg p\}} \\ \mathrm{QUD} & <\neg p ?>\oplus[\mathrm{Q}]\end{array}\right]\right.$

Enfin, le locuteur peut également nier une proposition qui n'a pas été assertée, mais qui est présente contextuellement, quand par exemple, elle justifie la question posée (cf. les exemples (24)-(25), et (27b) pour mica). Nous représentons cette proposition non assertée $q$ comme faisant partie de la partie privée du tableau de jeu ; plus précisément, c'est une proposition topique, qui est donc dans une relation de pertinence avec la question max-qud. Dans ce cas, la proposition non- $q$ est mise en discussion. Il reste une certaine incertitude en ce qui concerne le traitement de la question $p$ ? dans ce cas de figure. Nous maintenons sa présence dans QUD, de façon à rendre compte d'une continuation possible du dialogue sur le même thème, mais avec un changement dans les attendus qui président à la formulation des questions. 
(34) Tableau de jeu du locuteur quand il nie $q$ inférée contextuellement :

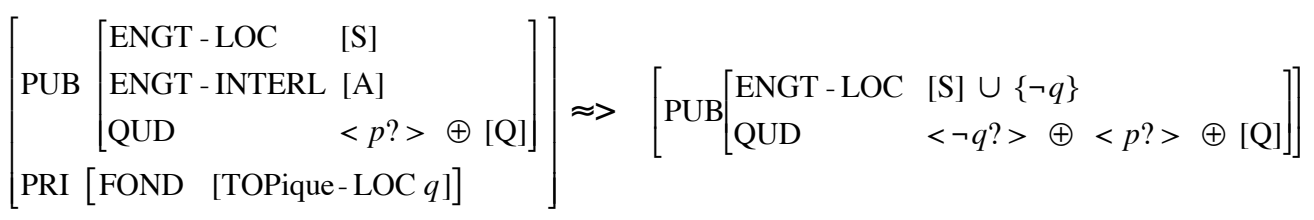

Il reste à relier la forme de la construction (4) à l'acte auquel elle correspond. D'abord, nous vérifions que cette construction n'est possible que dans une phrase racine, ce qui est attendu si elle est bien associée à un acte de langage comme la dénégation :
A. Sai chi Maria inviterà per il suo compleanno?
'Tu sais qui Maria va inviter pour son anniversaire?'
B. $\quad$ *Dovresti sapere che NESSUNO (non) inviterà
tu devrais savoir que personne NEG elle.invitera

Comme nous l'avons vu, le constituant initial en (4) n'a pas toujours la même fonction, et donc ne met pas toujours en jeu le même type de combinatoire. Les phrases de (4) partagent en revanche une propriété d'ordre des mots : elle mettent en jeu un constituant initial avec une prosodie spécifique. En HPSG, l'ordre des mots est représenté comme une liste de constituants (de signes) qui constitue la valeur du trait DOM. Nous laissons de côté l'étude de l'interface syntaxe-sémantique pour la négation en italien (voir Godard et Marandin 2006). Cependant, l'écriture précise de la contrainte requiert que nous disions quelle est notre analyse pour (4). Nous admettons que les mots-n dénotent des quantifieurs négatifs (neg-quant-rel), représentés comme des types d'objets scopaux, qui sont mis dans la valeur du trait STOCK, partagée par les constituants dominants jusqu'à ce qu'ils soient interprétés (voir de Swart et Sag 2002 pour le français). L'adverbe non n'est pas lui-même un quantifieur, mais c'est tout de même un objet scopal (neg-rel). Dans notre analyse, les négations de (4) ne sont pas interprétées avant le nœud qui domine le constituant initial (la phrase). Le constituant initial et le V (qui dominel'adverbe non, voir (8)), en tant que constituants, comportent donc la négation dans leur STOCK.

Nous représentons les assertions comme l'une des valeurs contextuelles des phrases racines. Elles sont modélisées comme un tableau de jeu dialogique, qui intègre les descriptions précédentes (28)-(34) : la description en input correspond à la valeur du trait DERNIER TOUR, et les éléments de la description en output se répartissent comme les éléments du tableau de jeu en cours. Les assertions sont des objets organisés en hiérarchie, comme on le voit en (36). Les constructions qui ont été étudiées dans ce texte sont des assertions de reprise. En particulier, les énoncés négatifs de (4) sont des dénégations, qui se répartissent en dénégations explicites et implicites, suivant que la proposition niée est explicite ou non. 
(36) Typologie des assertions (énoncés assertifs)

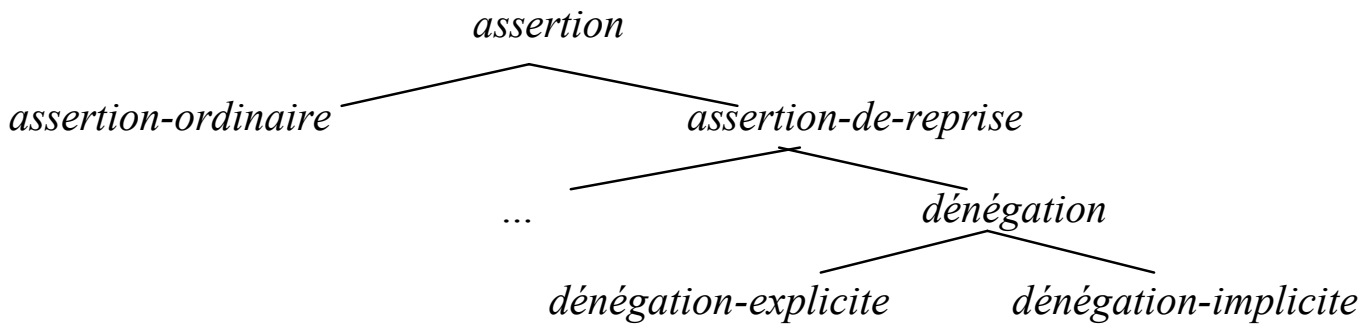

Nous avons donc l'implication (37) pour l'italien, qui décrit spécifiquement les énoncés comme ceux de (4). Le trait RACINE a une valeur booléenne ( \pm ), indiquant si la phrase est ou non une phrase racine ; les phrases racines sont soit directement dominées par la racine de l'arbre syntaxique représentant la structure en constituants de la phrase, soit un conjoint d'une coordination elle-même directement dominée par la racine de l'arbre.

Implication pour l'italien :

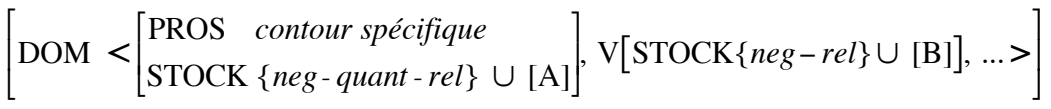

$$
\begin{aligned}
& =>\left[\begin{array}{ll}
\text { RACIN E } & + \\
\text { CONTEXTE } & \text { dénégation }
\end{array}\right]
\end{aligned}
$$

\section{Conclusion}

Nous avons avancé quatre hypothèses, relevant de niveaux distincts dans la grammaire, pour analyser les occurrences de non précédé d'une expression négative en italien. Premièrement, l'italien a deux stratégies de renforcement de la négation: soit il a recours à une expression non négative (ayant un effet de minimisation ou de généralisation), soit il recycle une expression négative. Deuxièmement, la négation renforcée est utilisée dans des énoncés de dénégation de proposition. On peut aussi faire une dénégation de proposition en utilisant la négation non renforcée, d'où les effets d'optionalité. Troisièmement, la négation renforcée apparaît dans une construction qui ne lui est pas propre. Cette construction est définie par un ordre des mots et un contour prosodique, et non pas par une structure syntaxique ou une articulation informationnelle particulière; de plus, ces deux propriétés communes existent également dans des constructions positives. Enfin, on a suggéré que le constituant initial marqué prosodiquement est distingué dans une dimension autre que la structure informationnelle : il signale la partie du contenu de l'énoncé qui est spécifiquement rejetée par le locuteur.

\section{Euvres citées}

Abeillé, Anne. 2006. Les nouvelles syntaxes, $2^{\circ}$ édition, Londres : Hermès.

Abeillé, Anne; D. Godard. 2000. French Word Order and Lexical Weight, In The Nature and Function of Syntactic Categories, R. Borsley (ed), 325-360. New York : Academic Press. Abeillé, Anne ; Danièle Godard. 2003. Les Prédicats Complexes dans les Langues Romanes, In 
Les Langues Romanes, D. Godard (ed),125-184. Paris: CNRS ED.

Abeillé, Anne ; Danièle Godard. 2006. La légèreté en français comme déficience de mobilité, Linguisticae Investigationes 29, K. Gerdes et C. Muller (eds), 11-24.

Acquaviva, Paolo. 1993. The Logical Form of Negation, PhD Dissertaion, Pisa, Scuola Normale Superiore [New York: Garland, 1997]

Benincà, Paola ; Gianpaolo Salvi ; Lorenza Frison. 1988. L'ordine degli elementi della frase e le costruzioni marcate, In Grande Grammatica di conzultazione, L. Renzi (ed), vol 1, 115215. Bologna : Il Mulino.

Bonami, Olivier; Danièle Godard. 2006. Lexical semantics and pragmatics of evaluative adverbs. MS, U. Paris 4 and U. Paris 7, à paraître in Christopher Kennedy et Louise Mc Nally, Dordrecht : Kluwer.

Büring Daniel. 1997. The Meaning of Topic and Focus: The 59th Street Bridge Accent. London: Routledge.

Carston, Robyn. 1996. Matalinguistic Negation and echoic use, Journal of Pragmatics 25, 309330 .

Chafe, Wallace. 1974, Language and consciousness, Language 50: 111-133.

Cinque, Guglielmo. 1976. Mica. Reprinted in G. Cinque, 1991, Teoria Linguistica e Sintassi Italiana. Bologna: Il Mulino.

Corblin, Francis ; Lucia Tovena. 2003. L'expression de la négation dans les langues romanes, In Les Langues Romanes, D. Godard (ed), 281-343. Paris: CNRS ED.

Dryer, Michael. 1996, Focus, pragmatic presupposition, and activated propositions, Journal of pragmatics $26,475-523$.

Espinal, Teresa. 2006. Licensing expletive negation and negative concord in Romance Languages. Conférence « La négation dans les langues romanes », Toulouse, février 2006. (CE VOLUME ??).

Floricic, Franck. 2005. La Négation dans les langues romanes, Lalies 25, 163-194.

Floricic, Franck ; Françoise Mignon. 2006. Négation et reduplication intensive en français et en italien. Conférence « La négation dans les langues romanes », Toulouse, février 2006. (CE VOLUME ??).

Geurts, Barts. 1998. The mechanisms of denial, Language $74: 274-307$.

Giannakidou, Anastasia. 2002. N-words and Negative Concord. MS, to appear in The linguistics Companion, H. van Riemsdijk et al (ed)., Oxford : Blackwell. (available on the web site for the encyclopedia).

Ginzburg, Jonathan. in prep., A semantics for interactions in dialogue, Stanford : CSLI and University of Chicago Press (ftp://ftp.cogsci.ed.ac.uk:pub/ginzburg).

Godard, Danièle ; Marandin Jean-Marie.2006. Reinfrocing negation : the case of Italian. In S. Müller (ed.) Proceedings of HPSG 06, on-line publication, 174-194. Stanford : CSLI.

Horn, Laurence. 1989. A Natural History of Negation. Chicago : U. of Chicago Press.

Jespersen, Otto. 1917. Negation in English and Other Languages. Copenhagen : Videnskabenes Selskab.

Kim, Jon-Bok. 2000. The Grammar of negation. Stanford : CSLI publ.

Kiparsky, P. and C. Condoravdi. 2006. Tracking Jespersen's cycle. in Proceedings of the 2nd International Conference of Modern Greek Dialects and Linguistic Theory, M. Janse, B. D. Joseph et A. Ralli (eds.). Mytilene: Doukas, 2006.

Laka, Itziar Murgaza. 1990. Negation in Syntax : on the nature of functional categories and projections, Thèse du MIT, Cambridge, Mass.

Manzotti, Emilio ; Alessandra Rigamonti.1991. La Negazione, in Grande Grammatica di consultazione, L. Renzi et G. Salvi (eds), vol 2, 245-317. Bologna: Il Mulino.

Marandin, Jean-Marie. 2005. Formatage de l'information: focus et contexte, in Interpréter en contexte, F. Corblin et C. Gardent, (eds). Londres: Hermes.

Pollard, Carl ; Ivan A. Sag. 1994. Head-driven Phrase Structure Grammar. Stanford : CSLI Publ. Et Chicago : Chicago University Press.

Prince, Ellen. 1981. Toward a taxonomy of given-new information, in Radical pragmatics, $\mathrm{P}$. Cole (ed), 223-255. New York : Academic Press.

Przepiorkowski, Adam. 1999. Negative Polarity Questions and Italian Negative Concord, Arbeitspapieren des SFB 340, U. Tübingen.

Rizzi, Luigi. 1982. Issues in Italian Syntax. Dordrecht: Foris Publ. 
Rizzi, Luigi. 1997. The fine structure of the Left Periphery, in Elements of Grammar: Handbook of Generative Grammar, L. Haegeman (ed), 281-304. Dordrecht : Kluwer.

Sag, Ivan A. ; Thomas Wasow ; Emily Bender. 2003. Syntactic Theory : a Formal Introduction, second ed. Stanford : CSLI Publ.

Van Der Sandt, Rob. 1991. Denial, Chicago Linguistics society, The parasession on negation, 331-344.

Stalnaker Robert. 1978 Assertion, In Pragmatics , P. Cole (ed.), 315-332. New-York : Academic Press.

Schwenter, Scott A. 2005. The pragmatics of negation in Brazilian Portuguese, Lingua 115 : 1427-1456.

Schwenter, Scott A. 2006. Fine Tuning Jespersen's cycle. To appear in a festschrift for L. Horn (available at the author's web site).

De Swart, H. ; I.A. Sag. 2002. Negation and Negative Concord in Romance. Linguistics and Philosophy 25, 373-417.

Tovena, Lucia. 2000. Neg-raising: negation as failure? In Perspective on Negation and Polarity Items, J. Hoeksmea, H. Rullman, V. Sanchez Valencia et T. van der Wouden (eds), 331356. Amsterdam : J. Benjamins.

Tseng, Jesse. 2002. Remarks on Marking, in Proceedings of the 8th international conference on $H P S G$, F. van Eynde L. Hellan et D. Beerman (sld). Stanford : csli Publ.

Vallduví Enric ; Maria Vilkuna. 1998. On Rheme and Kontrast. The Limits of Syntax, In Peter Culicover and Louise McNally (eds), 79-108. New-York: Academic Press.

Zanuttini, Raffaëlla. 1991. Syntatic Properties of Sentential Negation. A comparative study of Romance Languages. PhD dissertation, U. of Pennsylvania.

Zanuttini, Raffaëlla. 1997. Negation and Clausal Structure, a comparative study of Romance Languages. Oxford: Oxford University Press.

\section{Résumé}

La négation en italien est caractérisée par un contraste entre les positions préverbales et post-verbales : si un mot-n (nessuno, niente, nulla) apparaît avant le verbe, non n'apparaît pas ; si un mot-n apparaît après le verbe, non est obligatoire. En fait, il existe deux cas (avec une acceptabilité variable) où un mot-n pré-verbal co-existe avec non. Nous étudions le cas dans lequel le mot-n appartient au constituant initial de la phrase, qui est associé à une prosodie marquée. Nous montrons que la caractérisation de cette construction n'est pas à chercher du côté de la structure syntaxique, ni de la structure informationnelle, mais de la pragmatique. Ce type de phrase donne lieu à une énonciation appropriée si le locuteur nie une proposition accessible dans le contexte : il s'agit d'une négation métalinguistique (dénégation d'une proposition). La négation dans cet usage tend à utiliser des structures de négation renforcée, qui se fait donc soit par intégration d'indéfinis dans le système négatif, ce qui est le cas bien connu, soit par le recyclage de matériel négatif, comme la construction que nous étudions ici. Nous formalisons cette pragmatique dans un modèle du dialogue, qui utilise des structures de traits, et qui est susceptible de s'intégrer dans une grammaire HPSG.

\section{$\underline{\text { Abstract }}$}

Italian negation is said to be characterized by a contrast between n-words (nessuno, niente, nulla) in preverbal and postverbal positions. While non does not co-occur with preverbal n-words, it is obligatory with postverbal ones. In this paper, we study a construction, with speakers' variation, where preverbal n-words do co-occur with non: the n-word is part of the initial constituent which receives a marked prosody, anchored on its last word (which is not always the n-word). Instances of the construction have no other formal (syntactic or informational) properties in common. They constitute appropriate utterances for a speaker who denies an antecedent, contextually activated proposition. They realize one of the two formats by which metalinguistic negation tends to differentiate itself from descriptive, ordinary negation (via the integration of 
indefinites into the negative system, as is well-known, or via the recycling of negative material, with different syntactic constraints, as is the case here). We formalize our pragmatic approach in a model of dialogue, using feature structures, which can be part of a complete HPSG grammar.

Adresse des auteurs

Danièle Godard

CNRS, Laboratoire de Linguistique Formelle, UMR 7110

Université Paris 7, Case Postale 7031

2 Place Jussieu, Cedex 75251 Paris-Cedex 05

Jean-Marie Marandin

CNRS, Laboratoire de Linguistique Formelle, UMR 7110

Université Paris 7, Case Postale 7031

2 Place Jussieu, Cedex 75251 Paris-Cedex 05 